\title{
2.4 GHz Horn Antenna
}

\section{Goran Banjeglav, Krešimir Malarić}

This paper describes the building and testing of a $2.4 \mathrm{GHz}$ antenna which can be used for WLAN as well as for other purposes. The antenna was built to have highest gain at $2.4 \mathrm{GHz}$ although it can be used from frequency of $1.7 \mathrm{GHz}$ up to $2.6 \mathrm{GHz}$. The paper also describes the calculation of the antenna parameters and dimensions as well as the measurements of its parameters. After the numerical modeling and building, the antenna was tested in the laboratory.The numerical modeling was performed with XFDTD software and the testing of the antenna was done at the Microwave Laboratory of Faculty of Electrical Engineering and Computing, Zagreb. The results showed that the highest antenna gain of $9.46 \mathrm{~dB}$ was obtained at $2.437 \mathrm{GHz}$, which is a frequency used for wireless internet. The antenna can be used on ships in the port as well as on the sea for boosting the range and increasing the received power level of a wireless internet signal.

\section{KEY WORDS}

$\sim$ Horn antenna

$\sim$ Antenna gain

$\sim$ Numerical methods

$\sim$ Wireless internet

\section{INTRODUCTION}

For wireless signal transmission, it is necessary to have a sufficient signal level at the receiver end. WLAN (Wireless Local Area Network) usually operates on $2.4 \mathrm{GHz}$. This frequency is free to use (ISM - industrial, scientific, medical use) and therefore crowded by many technologies (Golmie and Mouveaux, 2001). An antenna with a high gain is often necessary to boost the signal level as well as to have constant connectivity. Today, there is a wide range of the available antennas at our disposal (Zentner, 1999; Balanis, 2005). There is also a possibility to purchase commercial antennas, but sometimes they do not suit the need of the user. Either the antenna gain is too small, or the frequency range of the antenna is not suited for our purpose. Sometimes, the commercial antennas are expensive as well, and building own antenna could be an option. Horn antenna with high gain is hard to find at most electronic equipment shops. Application of horn antenna include satellite communications, radio telescopes, radar systems and wi-fi.

Although horn antenna is a matter of a research for some time (Barrow and Chu, 1939), it is still a subject of research and improvements (Zang and Bergmann, 2014).

\section{ANTENNA DESIGN}

The first step in building the horn antenna is defining the necessary gain the antenna should have in the desired frequency range of operation. Antenna gain $(G)$ is defined as the ratio of the power transmitted in the direction of peak radiation and of an isotropic source (radiates equally in all directions). This ratio is 
usually expressed in $\mathrm{dB}$. A typical dipole antenna (used in many mobile phones for example) has antenna gain of $2.15 \mathrm{~dB}$. That means that the emitted power is boosted approximately by 65 $\%$ (3 dB would mean a $100 \%$ increase or double radiated power). The value of $2.15 \mathrm{~dB}$ sometimes is not enough, so in this paper the goal was set for $15 \mathrm{~dB}$ to be achieved with a horn antenna. The carrier frequency with maximum radiated power was selected to be $2.437 \mathrm{GHz}$, a 6th WLAN channel frequency.

Waveguide dimensions are determined depending on the frequency of use. Figure 1. shows the rectangular waveguide cross section, where $a$ is the width and $b$ is the height of the rectangular waveguide dimensions. Waveguide aperture should have dimensions equal to WR-430 standard (see Rectangular waveguide dimensions, 2014) giving $a=10.922 \mathrm{~cm}$ and $b=5.461$ $\mathrm{cm}$. Depth of waveguide (Figure 1 on the right) for frequency of $2.437 \mathrm{GHz}$ is equal to the half cut-off frequency wavelength $\lambda_{g}$ determined by (1), where $\lambda_{0}$ is the wavelength in free space and $\lambda_{c}$ is the wavelength of the cut-off frequency for the mode of transmission:

$\lambda_{g}=\frac{1}{\sqrt{\frac{1}{\lambda_{0}{ }^{2}}-\frac{1}{\lambda_{0}{ }^{2}}}}$

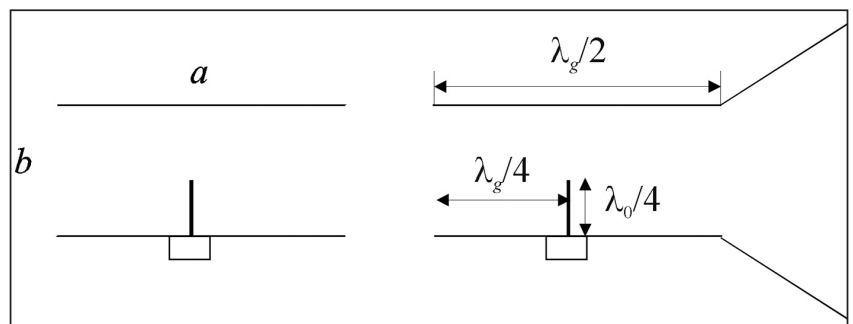

Figure 1.

Waveguide design.

Wavelength in free space $\lambda_{0}$ is determined by:

$\lambda_{0}=\frac{c}{f}=\frac{3 \cdot 10^{8} \mathrm{~m} / \mathrm{s}}{2.437 \cdot 10^{9} \mathrm{GHz}}=12.3 \mathrm{~cm}$

The higher order modes of transmission in a rectangular waveguide depend on its dimensions. The wavelengths of different modes are calculated by $\left(\lambda_{c}\right)_{m n}=\frac{2}{\sqrt{\left(\frac{m}{a}\right)^{2}+\left(\frac{n}{b}\right)^{2}}}$

where $m$ and $n$ are integer numbers. The first mode of transmission is $\operatorname{TE}_{10}(m=1, n=0)$, thus giving $\left(\lambda_{c}\right)_{10}=2 a=21.844$ $\mathrm{cm}$. By introducing $\lambda_{o}$ and $\lambda_{c}$ into (1), we obtain $\lambda_{g}=14.88 \mathrm{~cm}$. Therefore the depth of waveguide $\lambda_{g} / 2$ is equal to $7.44 \mathrm{~cm}$.

Next, it is necessary to determine the position of a feed antenna as well as its height. According to Figure 1. (right side), the distance of the feed antenna from the waveguide edge is equal to one quarter of a wavelength inside the waveguide, that is $\lambda_{g} / 4=3.72 \mathrm{~cm}$. The height of the feed antenna is equal to one quarter of the wavelength in a free space, that is, $\lambda_{0} / 4=3.075 \mathrm{~cm}$.

After the waveguide parameters are calculated, the next step is to determine the horn dimension. The horn will have pyramidal shape. The equation for calculating the antenna gain, $G$ is:

$G=\varepsilon_{a p} \cdot \frac{4 \Pi}{\lambda_{0}^{2}} \cdot A_{a p}$

where $\lambda_{0}$ is the wavelength, $\varepsilon_{a p}$ is the effective aperture (usually $0,51)$ coefficient and $A_{a p}$ is the area of aperture $(A \times B)$. For desired $G=15 \mathrm{~dB}$ (or $G=31,623$ ), it follows from (4) that $A_{a p}$ (that is $A x B$ ) $=0,07614$.

Figure 2. shows the horn antenna design and required dimension of the antenna. The following equations are valid for rectangular pyramidal waveguide (see High performance horn antenna design (II), 2014):

$A \cdot B=0.07614$

$\rho_{H}\left(1-\frac{a}{A}\right)=\rho_{E} 1-\left(\frac{b}{B}\right)=R_{H}=R_{E}$

$A=\sqrt{3 X a_{H}}$

$$
B=\sqrt{2 \lambda \rho_{E}}
$$




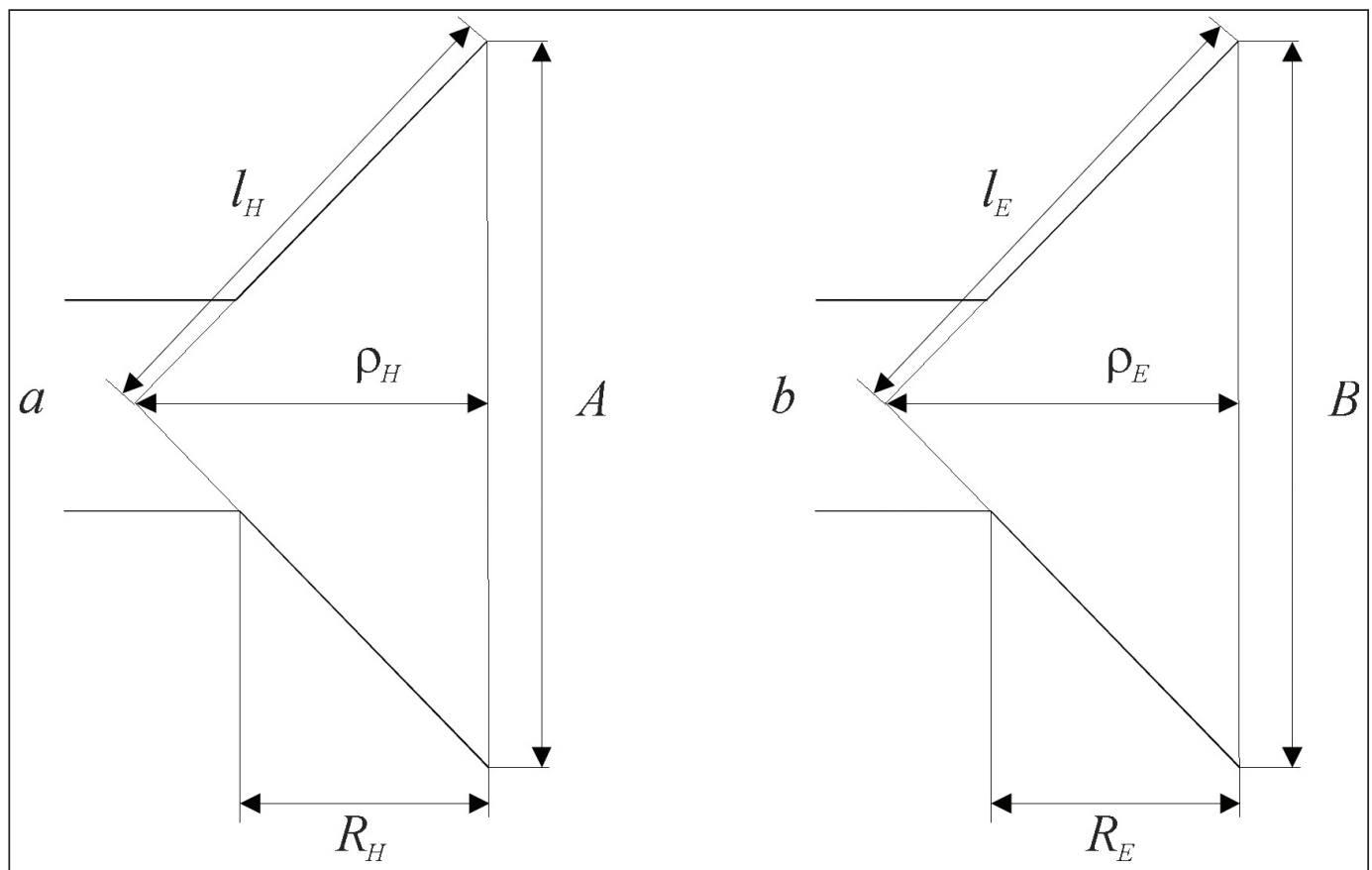

Figure 2.

Horn antenna design.

Thus, we have system of four equations with four unknowns $(A$, $\left.B, \rho_{H^{\prime}} \rho_{E}\right)$. The results of calculation are given in Table 1 . In order to determine the remaining dimensions, the following equations

$I_{g}=\sqrt{\rho_{H}^{2}+\left(\frac{A}{2}\right)^{2}}$ will be used:

$\rho_{H} / R_{H}=A /(A-a)$

$\rho_{E} / R_{E}=B /(B-b)$

$$
I_{E}=\sqrt{\rho_{E}^{2}+\left(\frac{B}{2}\right)^{2}}
$$

Table 1.

Antenna dimensions.

$\begin{array}{lllllllll}A[\mathrm{~m}] & B[\mathrm{~m}] & a[\mathrm{~m}] & b[\mathrm{~m}] & \rho_{H}[\mathrm{~m}] & \rho_{E}[\mathrm{~m}] & R_{E}=R_{H}[\mathrm{~m}] & I_{H}[\mathrm{~m}] & I_{E}[\mathrm{~m}] \\ 0.321 & 0.242 & 0.1103 & 0.0557 & 0.272 & 0.232 & 0.1796 & 0.2074 & 0.2015\end{array}$




\section{SIMULATION MODEL}

Horn antenna was simulated using the XFDTD software (Remcom, 2006). Figures 3 and 4 are showing the simulation results of a 3D radiation pattern in space, while Figure 5 shows $2 \mathrm{D}$ radiation pattern and gain in polar coordinate system.

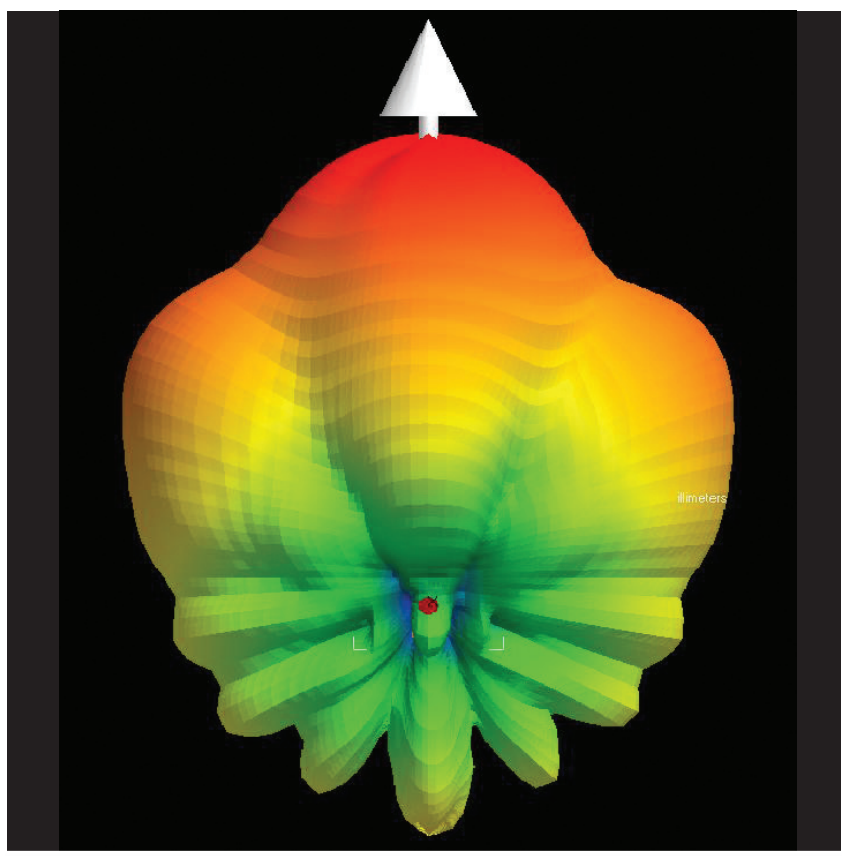

Figure 3.

$3 \mathrm{D}$ radiation pattern.

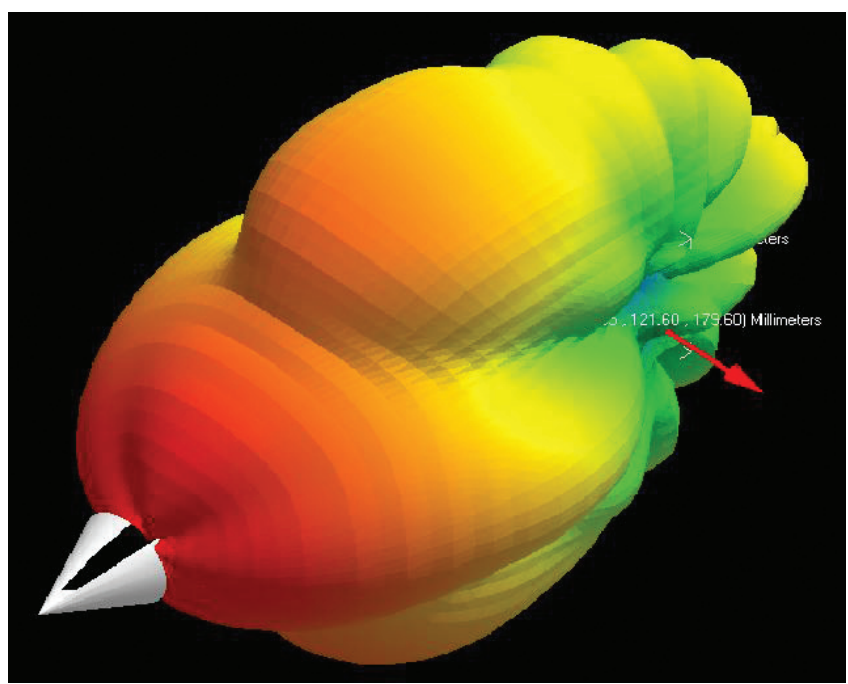

Figure 4.

$3 \mathrm{D}$ radiation pattern from a different angle.
It can be seen from Figure 5 that modeled antenna has the desired characteristics, that is, the gain of $15 \mathrm{~dB}$ at an angle of $0^{\circ}$. The gain stays constant up to $\pm 10^{\circ}$ on each side from the direction of maximum gain. On higher angles the gain drops to about $5 \mathrm{~dB}$. The directivity of the antenna is not high because main lobe is quite wide and there are several side lobs present.

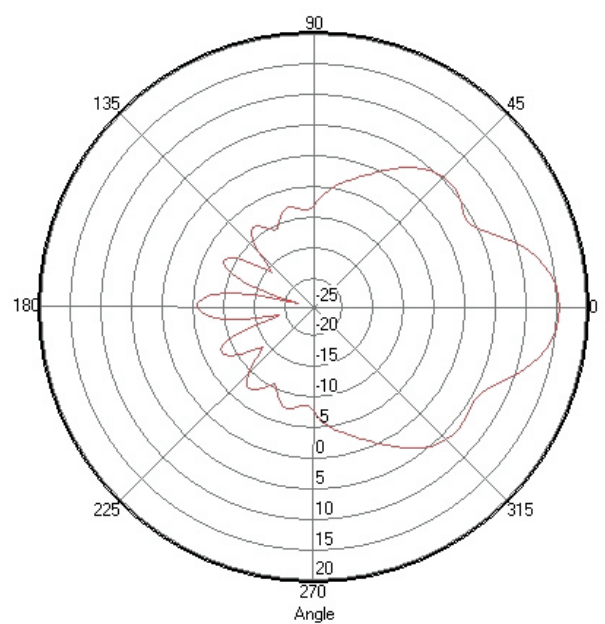

Figure 5 .

2D horizontal radiation pattern and gain.

\section{MEASUREMENTS}

Parameter measurements were performed at the Microwave Laboratory of the Department of Radiocommunications, Faculty of Electrical Engineering and Computing, University of Zagreb. Measurement set-up is shown in Figure 6. The distance of $7 \mathrm{~m}$ between the antenna and the spectrum analyzer was chosen due to the laboratory size dimensions. For generator we have used was HP 8350B and for the spectrum analyzer NARDA SRM 3000 was utilized. Horn antenna, made out of copper $0.55 \mathrm{~mm}$ thick and with a $N$ type connector, was connected to the generator using RG 213 cable.

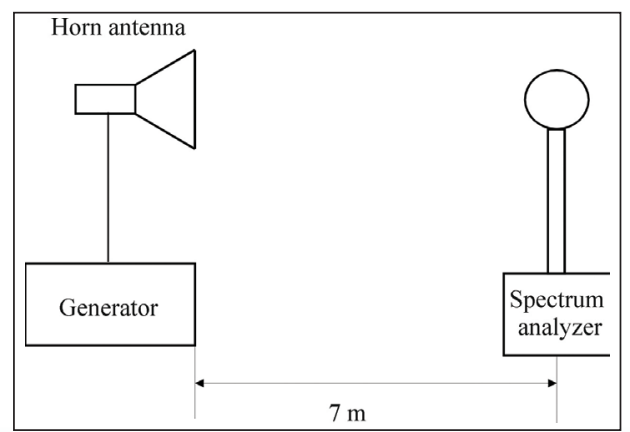

Figure 6.

Measurement set-up 
The measurements included measuring power density vs frequency, calculating propagation losses and measuring horn antenna gain compared to the dipole antenna at the frequency of $2.4 \mathrm{GHz}$.
The measurements were performed in the frequency range from $1.7 \mathrm{GHz}$ to $2.6 \mathrm{GHz}$. The measured power density $\left(P_{D}\right)$ results are shown in Figure 7. It can be seen that the received power density is highest at app $2.4 \mathrm{GHz}$, and then it drops to $2.6 \mathrm{GHz}$.

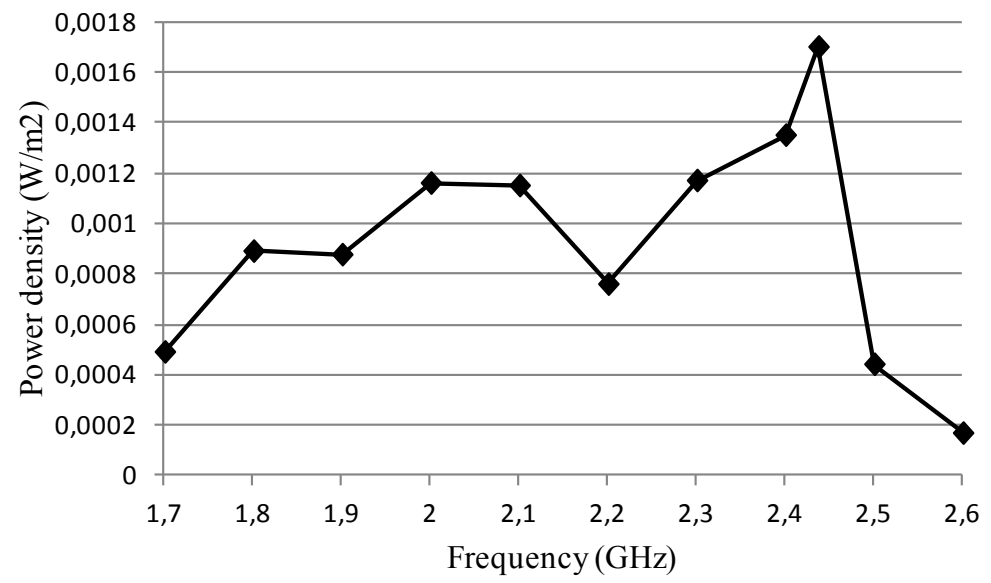

Figure 7.

Power density $\left(P_{D}\right)$ vs frequency.

Antenna gain $\left(G_{t}\right)$ is calculated from

$G_{t}=P_{r}-P_{t}-G_{r}-L$

where $P_{r}$ and $P_{t}$ are transmitted and received power, $G_{r}$ is the receiver antenna gain and $L$ is the signal attenuation. Signal attenuation $L$ depends on the frequency $(\lambda=c / f)$ and the distance $d$ between the antennas and can be calculated from

$L=20 \log \left(\frac{\lambda}{4 \Pi d}\right)$

The values of $L$ are given in Table 2. Transmitted power $P_{t}$ was set to be $+15 \mathrm{dBm}$.

Table 2.

Measurement results of received power, attenuation and gain.

\begin{tabular}{|c|c|c|c|}
\hline Frequency [GHz] & Received power $P_{r}[\mathrm{dBm}]$ & Signal attenuation $L$ [dB] & Antenna Gain $G_{t}[d B]$ \\
\hline 1.7 & -29.13 & 53.95 & 4.07 \\
\hline 1.8 & -27.04 & 54.44 & 6.67 \\
\hline 1.9 & -27.58 & 54.91 & 6.59 \\
\hline 2.0 & -27.89 & 55.36 & 6.72 \\
\hline 2.1 & -27.91 & 55.78 & 7.13 \\
\hline 2.2 & -29.48 & 56.19 & 5.96 \\
\hline 2.3 & -27.99 & 56.57 & 7.84 \\
\hline 2.4 & -27.72 & 56.94 & 8.48 \\
\hline 2.437 & -26.86 & 57.08 & 9.46 \\
\hline 2.5 & -32.94 & 57.30 & 3.61 \\
\hline 2.6 & -36.89 & 57.64 & 0.00 \\
\hline
\end{tabular}


Received power (with $G_{r}=1$, because it is embedded in the value of received power by spectrum analyzer) is calculated from

$P_{r}=\frac{P_{D}}{4 \cdot \Pi} \cdot \frac{C^{2}}{f^{2}} G_{r}$

where $P_{D}$ is the measured power density, $c$ is the speed of light and $f$ is the frequency.

The values of $P_{r}$ are given in Table 2. Introducing values from (14) and (15) into (13), antenna gain $\left(G_{t}\right)$ can be calculated.

We must take into calculation additional losses for indoor propagation $(1 \mathrm{~dB} / \mathrm{m})$, losses in the cable $(0.25 \mathrm{~dB} / \mathrm{m})$ and connector losses ( $0.5 \mathrm{~dB}$ each). Final results for horn antenna gain (Gt) are given in Table 2. The frequency dependence of the antenna gain is shown in Figure 8. It can be seen that the gain at frequency $2.437 \mathrm{GHz}$ is highest and equal to $9.46 \mathrm{~dB}$. This value is less than $15 \mathrm{~dB}$, the value which was hoped for.

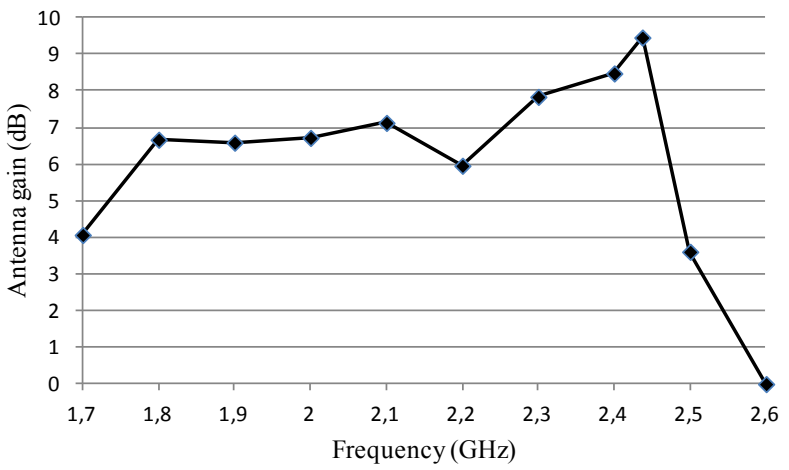

Figure 8.

Antenna gain $\left(G_{t}\right)$ vs frequency.

The difference can be result of a measurement error or material deformations. Thicker metal would probably result in a higher antenna gain (the geometry of the antenna would be more stable). However, antenna gain stays above $6 \mathrm{~dB}$ in almost entire frequency range of interest.

For verification of the measurement method and the results, horn antenna gain was compared to the dipole antenna at frequency of $2.4 \mathrm{GHz}$ with its known value of gain being 2.15 $\mathrm{dB}$. The above mentioned measured method gave the result for the dipole antenna gain to be $2.29 \mathrm{~dB}$ which meant a measuring error of only $0.14 \mathrm{~dB}$.

\section{CONLUSION}

The $2.4 \mathrm{GHz}$ horn antenna was built based on the XFDTD simulation model. The designed antenna can be used for WLAN access on ships as well as for other purposes. The antenna gain of app. $9.5 \mathrm{~dB}$ is much higher than dipole antenna $(2.15 \mathrm{~dB})$ which is normally used. The desired antenna gain of $15 \mathrm{~dB}$ could be achieved with a more precise building and thicker metal instead of $0.55 \mathrm{~mm}$ copper which was used in our case. Although intended for $2.4 \mathrm{GHz}$, the antenna can be used in the frequency range from $1.7 \mathrm{GHz}$ to $2.6 \mathrm{GHz}$ with a high gain.

\section{REFERENCES}

Balanis, C., (2005), Antenna Theory: Analysis and Design, 3rd Edition, Hoboken: Wiley-Interscience.

Barrow, W. L., Chu, L. J., (1939), Theory of Electromagnetic Horn, Proceedings of IRE, 27 (1), pp. 51-64.

http://dx.doi.org/10.1109/JRPROC.1939.228693

Golmie, N; Mouveaux, F., (2001), Interference in the $2.4 \mathrm{GHz}$ ISM Band: Impact on the Bluetooth Access Control Performance, Proc. IEEE International Conference on Communications, Helsinki, Finland, June 11-14, pp. 2540-2545.,

http://dx.doi.org/10.1109/ICC.2001.936608

High performance horn antenna design (II), available at:

http://www.radio.feec.vutbr.cz/kosy/soubory/bocia/High_performance_horn_ antenna design_II.pdf, [accessed 15 July 2014.].

Rectangular waveguide dimensions, available at:

http://www.microwaves101.com/encyclopedia/waveguidedimensions.cfm, [accessed 06 June 2014.].

Remcom, (2006), Full-wave, 3D, Electromagnetic Analysis Software Reference Manual, version 6.4, Remcom inc.

Zang, S. R., Bergmann, J. R., (2014), Analysis of Omni directional Dual-Reflector Antenna and Feeding Horn Using Method of Moments, IEEE Transactions on Antennas and Propagation, 62(3), pp. 1534-1538.

http://dx.doi.org/10.1109/TAP.2013.2296775

Zentner, E., (1999), Antene i radiosustavi, Zagreb: Graphis. 